\title{
Excited State Properties of Oligophenyl and Oligothienyl Swivel Cruciforms
}

\author{
J. Pina,$^{\dagger}$ J. Seixas de Melo, ${ }^{*}, \dagger$ H. D. Burrows, ${ }^{\dagger}$ F. Galbrecht, ${ }^{\ddagger}$ A. Bilge, ${ }^{\ddagger}$ C. J. Kudla, ${ }^{\ddagger}$ and \\ U. Scherf \\ Department of Chemistry, University of Coimbra, 3004-535 Coimbra, Portugal, and Makromolekulare Chemie, \\ Bergische Universität Wuppertal, Gaußstr. 20, 42097 Wuppertal, Germany
}

Received: September 12, 2007; In Final Form: October 23, 2007

\begin{abstract}
A comprehensive study has been undertaken of the electronic spectral and photophysical properties of two oligophenyl (BPH and BPHF) and one oligothienyl (BTF) swivel cruciforms involving measurements of absorption, fluorescence, and phosphorescence spectra, quantum yields of fluorescence $\left(\phi_{\mathrm{F}}\right)$, phosphorescence $\left(\phi_{\mathrm{Ph}}\right)$ and triplet formation $\left(\phi_{\mathrm{T}}\right)$, lifetimes of fluorescence $\left(\tau_{\mathrm{F}}\right)$ and of the triplet state $\left(\tau_{\mathrm{T}}\right)$, and quantum yields of singlet oxygen production $\left(\phi_{\Delta}\right)$. From these, all radiative $k_{\mathrm{F}}$ and radiationless rate constants, $\mathrm{k}_{\mathrm{IC}}$ and $\mathrm{k}_{\mathrm{ISC}}$, have been obtained in solution. The energies of the lowest lying singlet and triplet excited states were also determined at $293 \mathrm{~K}$. Several of the above properties have also been obtained at low temperature and in the solid state (thin films). In general, for the phenyl oligophenyl (BPH) and for the oligothienyl (BTF) compounds, the radiationless decay channels $\left(\phi_{\mathrm{IC}}+\phi_{\mathrm{ISC}}\right)$ are the dominant pathway for the excited-state deactivation, whereas with the fluorene based oligophenyl BPHF the radiative route prevails. In contrast to the general rule found for related oligomers (and polymers) where radiative emission from $\mathrm{T}_{1}$ is absent, with the compounds studied, phosphorescence has been observed for all of the compounds, indicating that this type of functionalization can lead to emissive triplets. Time-resolved fluorescence decays with picosecond resolution revealed multiexponential (bi- and triexponential) decay laws compatible with the existence of more than one species or conformation in the excited state. These results are discussed on the basis of conformational flexibility in the excited state.
\end{abstract}

\section{Introduction}

While many aspects of the design and synthesis of conjugated organic polymers for use in light emitting and other optoelectronic devices have already been extensively studied and while a number of successful systems are already being commercialized, for future applications within these areas, it is likely that the solid state conformation and morphology of these $\pi$-conjugated systems will rule the efficiency of the devices. The degree of crystallinity is a fundamental factor, and for preparation of light emitting devices, it is often advantageous to employ amorphous material, since these minimize interchain interactions and aggregates, which tend to promote nonradiative recombination processes. ${ }^{1}$ There is, therefore, a need for rational design and study of new solution processable photonic materials which will hinder crystallization and form highly luminescent solid films.

In a recent publication, the synthesis of $2,5,2^{\prime}, 5^{\prime}$-tetra(4terbutylphenyl)-1,1'-biphenyl as the prototype of the oligophenyl swivel cruciform family has been reported. ${ }^{1}$ One of the main goals of that study was to see whether this molecular design could be advantageous for solution-phase fabrication of ordered thin films for incorporation in a diverse range of optoelectronic devices. Since phenylene polymeric systems are difficult to study and to process in solution, initial studies have concentrated on oligomers. ${ }^{2}$ Unfortunately, many of these oligomeric systems also display low solubility upon increasing chain length. Recently, the application of swivel cruciform architecture to

* Corresponding author. E-mail: sseixas@ci.uc.pt. Fax: 351239827703.

$\dagger$ University of Coimbra.

$\doteqdot$ Bergische Universität Wuppertal. $\pi$-conjugated oligomers has gained significant interest because they can be considered to be structures where the band gap between the highest occupied molecular orbital (HOMO) and the lowest unoccupied molecular orbital (LUMO) and consequently the electronic properties of the oligomers can be tuned, ${ }^{3-5}$ also because of their compact structure compared with the linear analogues and the fact that the single bond between the oligomers chains permits a certain degree of rotation of the arms in the cruciform, which can lead to increased solubility. ${ }^{6}$ The amorphous character of organic thin films prevents grain boundaries (crystallites) to be formed during film preparation leading to spatial homogeneity of the charge carriers transport properties. $^{7}$ In the solid state, it was found that the cruciform 2,5,2',5'-tetra(4-terbutylphenyl)-1,1'-biphenyl molecular structure adopts a folded helical conformation, characterized by an almost parallel orientation of the terminal phenyl rings combined by a partial overlap. ${ }^{1}$ This type of conformation should enhance charge mobility since it will promote extended $\pi-\pi$ orbital overlap.

In the present study, we focus on the emission properties of this family of oligocruciforms. In addition to the study of the optical properties of the former cruciform oligomer possessing a biphenyl central unit, two additional oligoaryl cruciforms with 9,9'-dialkylfluorenyl extended arms and with 2,5-thienyl central core were also investigated. The study involves the characterization of the spectral and photophysical properties of these oligocruciforms in solution and in the solid state (thin films). Time-resolved fluorescence decays in the picosecond domain have revealed that the cruciforms here investigated display a complex behavior which is compatible with conformational changes in the excited state. 


\section{Experimental Section}

The synthetic procedures toward the cruciform dimer BPH have been described elsehwere. ${ }^{1}$ Detailed synthesis procedures and characterization data for BPHF and BTF can be found in Supporting Information.

All of the solvents used were of spectroscopic or equivalent grade. For the absorption and emission experiments in methylcyclohexane $(\mathrm{MCH})$ and 3-methylpentane (3MP), the concentrations of the solutions ranged from $1 \times 10^{-5}$ to $10^{-6} \mathrm{M}$.

Absorption and fluorescence spectra were recorded on Shimadzu UV-2100 and Horiba-Jobin-Ivon SPEX Fluorog 3-22 spectrometers, respectively. Fluorescence spectra were corrected for the wavelength response of the system. Fluorescence quantum yields were measured using bithiophene $\left(\phi_{\mathrm{F}}=0.014\right)$ and terthiophene $\left(\phi_{\mathrm{F}}=0.057\right)$ as standards. ${ }^{8}$

Nanosecond fluorescence decays were measured using a home-built time-correlated single-photon counting (TCSPC) apparatus with an IBH NanoLED (339 or $373 \mathrm{~nm}$ ) excitation source. ${ }^{9}$ Fluorescence decay times with picosecond time resolution were obtained in an apparatus described elsewhere ${ }^{10}$ except that a new Becker \& Hickl SPC-630 board was used as a TCSPC device. Full details have previously been reported, and the time resolution of the equipment is approximately 3 ps. ${ }^{11,12}$ Alternate measurements $(1000 \mathrm{cpc})$ of the pulse profile at the excitation wavelength and the sample emission were performed until $5 \times 10^{4}$ counts at the maximum were reached. Decays were analyzed using the modulating functions method of Striker with automatic correction for the photomultiplier "wavelength shift". ${ }^{13}$

The experimental setup used to obtain triplet state absorption spectra and quantum yields involves an Applied Photophysics laser flash photolysis apparatus pumped by a Nd:YAG laser (Spectra Physics), as described in detail elsewhere. ${ }^{9}$ Transient spectra were obtained by monitoring the optical density change at intervals of $5-10 \mathrm{~nm}$ over the $300-850 \mathrm{~nm}$ range and averaging at least 10 decays at each wavelength. First-order kinetics were observed for the decay of the lowest triplet state. Excitation was at $266 \mathrm{~nm}$ or $355 \mathrm{~nm}$ with an unfocused beam. Special care was taken in determining triplet yields to have optically matched dilute solutions ( $\mathrm{abs} \approx 0.2$ in a $10 \mathrm{~mm}$ square cell) and low laser energy $(\leq 2 \mathrm{~mJ})$ to avoid multiphoton and triplet-triplet $(\mathrm{T}-\mathrm{T})$ annihilation effects.

The triplet molar absorption coefficients were determined by the energy transfer method, ${ }^{14}$ using naphthalene, $\epsilon_{\mathrm{T}}=24500$ $\mathrm{M}^{-1} \mathrm{~cm}^{-1}(415 \mathrm{~nm})^{15}$ as triplet energy donor. Solutions of cruciform oligomers $\left(2 \times 10^{-5} \mathrm{~mol} \mathrm{dm}{ }^{-3}\right)$ were dissolved in methylcyclohexane solutions of naphthalene $\left(\lambda_{\mathrm{exc}}=266 \mathrm{~nm}\right)$ $10^{-3} \mathrm{~mol} \mathrm{dm}^{-3}$. All solutions were degassed with argon for $\approx 20$ min and sealed. The molar triplet-triplet molar absorption coefficients were then determined from eq $1:^{14}$

$$
\frac{\epsilon_{\mathrm{TT}}^{\mathrm{D}}}{\epsilon_{\mathrm{TT}}^{\mathrm{A}}}=\frac{\Delta \mathrm{OD}^{\mathrm{D}}}{\Delta \mathrm{OD}^{\mathrm{A}}}
$$

where $\epsilon_{\mathrm{TT}}^{\mathrm{D}}$ and $\epsilon_{\mathrm{TT}}^{\mathrm{A}}$ are the triplet molar absorption coefficients of donor and acceptor respectively; $\Delta \mathrm{OD}^{\mathrm{D}}$ is the maximum absorbance from the transient triplet-triplet absorption spectra of the donor in the absence of acceptor; $\Delta \mathrm{OD}^{\mathrm{A}}$ is the maximum absorbance of the acceptor triplet when both the donor and the acceptor are present. When the acceptor decay rate constant $\left(k_{3}\right)$ is not negligible, corrections were made for determination of $\Delta \mathrm{OD}^{\mathrm{A}}$ using eq $2,{ }^{14}$

$$
\Delta \mathrm{OD}_{\mathrm{obs}}^{\mathrm{A}}=\Delta \mathrm{OD}^{\mathrm{A}} \exp \left[-\frac{\ln k_{2} / k_{3}}{k_{2} / k_{3}-1}\right]
$$

where $k_{2}$ is the donor decay rate constant in the presence of acceptor and $\Delta \mathrm{OD}_{\mathrm{obs}}^{\mathrm{A}}$ is taken from the maximum observed in the triplet-singlet difference spectra of the acceptor in the presence of donor.

The intersystem crossing yields for the compounds $\left(\phi_{\mathrm{T}}^{\mathrm{cp}}\right)$ were obtained by comparing the $\triangle \mathrm{OD}$ at $525 \mathrm{~nm}$ of benzene solutions of benzophenone or the $\Delta \mathrm{OD}$ at $415 \mathrm{~nm}$ of ethanol solutions of naphthalene (standards) optically matched (at the laser excitation wavelength) and of the compound using the equation: ${ }^{16,17}$

$$
\phi_{\mathrm{T}}^{\mathrm{cp}}=\frac{\epsilon_{\mathrm{TT}}^{\text {benzophenone }}}{\epsilon_{\mathrm{TT}}^{\mathrm{cp}}} \cdot \frac{\Delta \mathrm{OD}_{\max }^{\mathrm{cp}}}{\Delta \mathrm{OD}_{\max }^{\text {benzoph. }}} \cdot \phi_{\mathrm{T}}^{\text {benzophenone }}
$$

Room-temperature singlet oxygen phosphorescence was detected at $1270 \mathrm{~nm}$ using a Hamamatsu R5509-42 photomultiplier, cooled to $193 \mathrm{~K}$ in a liquid nitrogen chamber (products for research model PC176TSCE-005), following laser excitation of aerated solutions at $266 \mathrm{~nm}$ or $355 \mathrm{~nm}\left(\mathrm{OD} @ \lambda_{\mathrm{exc}}=0.20\right)$, with an adapted Applied Photophysics flash kinetic spectrometer, as reported elsewhere. ${ }^{9} 1 H$-Phenalen-1-one (perinaphthenone) in toluene $\left(\phi_{\Delta}=0.93, \lambda_{\text {exc }}=355 \mathrm{~nm}\right)$ and biphenyl in cyclohexane $\left(\phi_{\Delta}=0.73, \lambda_{\text {exc }}=266 \mathrm{~nm}\right)$ were used as standard. ${ }^{15}$

Thin films from the compounds were obtained with a DeskTop Precision Spin Coating System, model P6700 series from Speedline Technologies. The solid-state thin film from the samples were obtained by deposition of a few drops of solution onto a circular sapphire substrate $(10 \mathrm{~mm}$ diameter $)$ followed by spin coating (2500 rpm) in a nitrogen saturated atmosphere (2 psi). Solutions for spin-coating were prepared by adding 2 $\mathrm{mg}$ of the samples to $15 \mathrm{mg}$ of Zeonex in $200 \mu \mathrm{L}$ toluene solution with stirring at $40{ }^{\circ} \mathrm{C}$ for $30 \mathrm{~min}$.

Fluorescence emission spectra of the thin films were obtained with a Horiba-Jobin-Yvon integrating sphere. The solid-state photoluminescence quantum yields in thin films were obtained with this integrating sphere, using the method outlined by de Mello et al. ${ }^{18}$ and developed by Palsson and Monkman ${ }^{19}$ using a setup similar to ours. The following equation was used to determine the fluorescence quantum yields, eq 4 ,

$$
\begin{aligned}
& \phi_{\mathrm{F}}^{\text {solid }}= \\
& \frac{\int^{\text {sample }} I(\lambda) \mathrm{d} \lambda}{\left(\int^{\text {scatter alone }} I(\lambda) \mathrm{d} \lambda-\int^{\text {scatter in sample }} I(\lambda) \mathrm{d} \lambda\right) \times 10^{\Delta \mathrm{OD}\left(\lambda_{\text {exc }}\right)}}
\end{aligned}
$$

where $\phi_{\mathrm{F}}^{\text {solid }}$ is the fluorescence quantum yield for the cruciform oligomer in a thin-film, $\int$ sample $I(\lambda) \mathrm{d} \lambda$ is the integrated area under the emission of the cruciform in the thin-film (which excludes the integration of Rayleigh peak), $\int$ scatter alone $I(\lambda) \mathrm{d} \lambda$ is the integrated area under the Rayleigh peak of a sample containing only the sapphire support, and $\int$ scatter in sample $I(\lambda) \mathrm{d} \lambda$ is the integrated area under the Rayleigh peak in the emission spectra of the cruciform oligomer in the thin film. Since in practice the emission from the sample is much weaker than the scattered excitation light (the Rayleigh peak), the spectra is recorded with a filter that attenuates the emission intensity at 


\section{SCHEME 1}

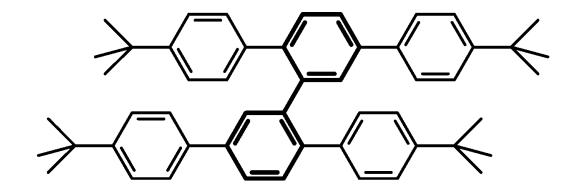

BPH
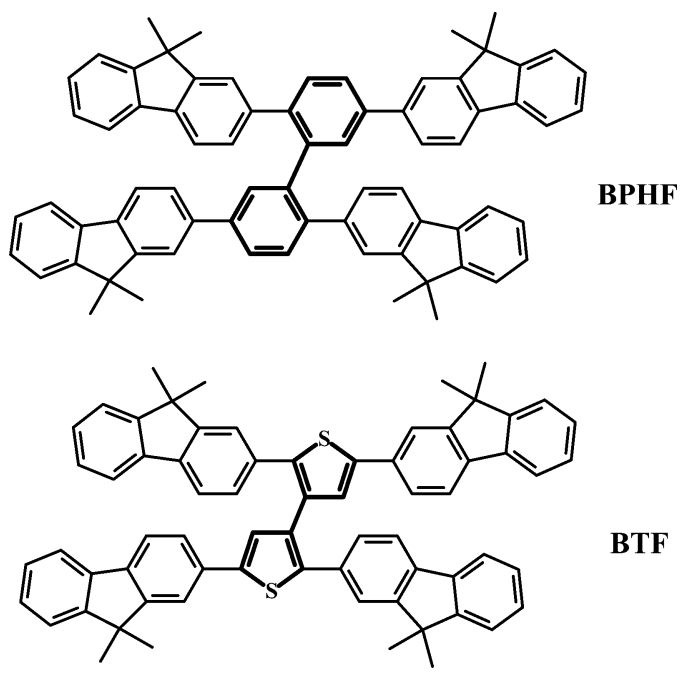

BTF

the excitation wavelength. This is considered in eq 4 with $10^{\Delta \mathrm{OD}\left(\lambda_{\text {exc }}\right)}$, the filter transmittance at the excitation wavelength.

\section{Results and Discussion}

The structures and acronyms of the oligophenyl and oligothienyl swivel cruciforms studied are shown in Scheme 1. These involve cruciform oligomers with biphenyl (BPH and BPHF) or bithiophene (BTF) central cores and possess 4-tert-butylphenyl or 9,9-dimethylfluorene side branches. BPH possesses an identical central unit to BPHF but a different backbone structure (see Scheme 1). These structural modifications are aimed at improving the oligomer solubility and at minimizing crystallization. ${ }^{1}$

Electronic Absorption and Luminescence Spectra. Figure 1 presents the absorption (full lines) and fluorescence spectra (dashed lines) of these compounds studied in solution at room temperature (RT, $293 \mathrm{~K})$, low temperature $(77 \mathrm{~K})$, and in the solid state (thin films). The absorption spectra for these samples are broad and devoid of vibronic structure. Substitution of the 4-tert-butylphenyl (BPH) side chains with 9,9-dimethylfluorene (BPHF) red shifts the absorption maximum in solution by $\approx 47$ $\mathrm{nm}$. The replacement of the central biphenyl core (BPHF) with a bithiophene unit $(\mathrm{BTF})$ also leads to a red shift $(\approx 40 \mathrm{~nm})$ in the absorption spectra.

No significant changes are observed in the shape of the absorption band or the wavelength maxima either upon cooling to $77 \mathrm{~K}$ or with samples in thin films at RT.

In contrast to what is observed with the absorption spectra (marked red shift of the BPHF spectra relative to $\mathrm{BPH}$ ), the fluorescence spectra of BPHF and BPH in solution at $293 \mathrm{~K}$ are virtually identical in terms of both band shape and maxima (see Table 1 and Figure 1).

Upon cooling, enhancement of the vibronic structure in the emission spectra of BPHF and BTF was observed. The low temperature $(77 \mathrm{~K})$ solution spectra of $\mathrm{BPH}$ and $\mathrm{BPHF}$ are blueshifted compared with those at room temperature. The opposite behavior was seen for BTF, where a bathochromic shift of $\approx 5$ nm was observed.

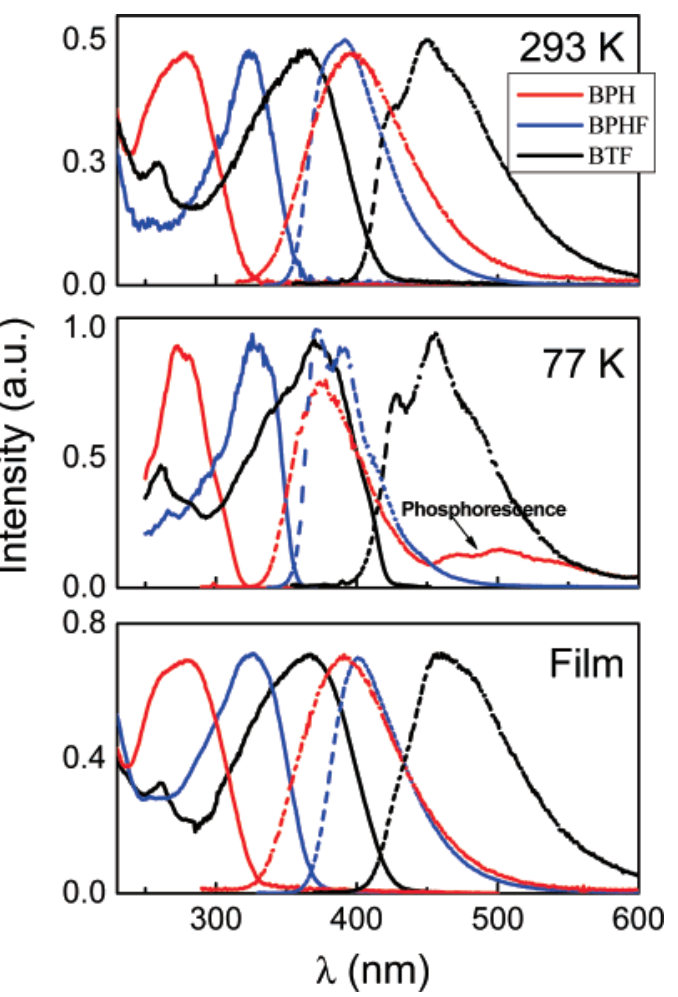

Figure 1. Absorption (full lines) and fluorescence emission spectra (dashed lines) for the cruciform oligomers in solution at room (293 K) and low temperature $(77 \mathrm{~K})$ and in the solid state (thin films).

In the solid state, the emission spectra for BPHF and BTF are $\approx 10 \mathrm{~nm}$ red-shifted in comparison with the solution data at room temperature. In contrast, with $\mathrm{BPH}$, the fluorescence emission band presents a hypsochromic shift of approximately $4 \mathrm{~nm}$. These minor differences in the emission reflect changes in environment on going from solution to thin films. ${ }^{1}$

Time-Resolved Behavior. Fluorescence lifetimes were also obtained in solution and in the solid state and initial studies (with time-resolution of $\approx 150 \mathrm{ps}$ ) suggested single-exponential decays for all compounds (see Table 2). However, when the solution decays are investigated with picosecond time resolution $(\sim 3 \mathrm{ps})^{11}$ the decays are now multiexponential (bi- and triexponential) following decay laws according to eq 5;

$$
I(t)=\sum_{i j} a_{i} \mathrm{e}^{-t / \tau_{j}}
$$

where $a_{i}(i=1,2$, for BPH and BPHF and $i=1,2,3$ for BTF $)$ are the pre-exponential factors and $\tau_{j}$ are the decays times with $j=2$ for BPH and BPHF and $j=3$ for BTF. It can be seen from Figure 2 that for BPHF and BTF the shortest decay time appears as a rise time. This indicates that the component associated with this decay time is formed in the excited state at the expense (or during the decay) of other species. In fact, although this has not been studied exhaustively in this work, it is likely that at shorter wavelengths this component would appear to be associated with a positive pre-exponential factor. An example of this is found with $\mathrm{BPH}$, when the decays are collected at different emission wavelengths $\left(\lambda_{\mathrm{em}}\right)$, Figure 3 . In this case (see Figure 3), the decay times do not show any significant change along the fluorescence spectra and can be considered to be independent of the $\lambda_{\mathrm{em}}$, whereas the preexponential factors change significantly, in agreement with a progressive spectral shift.

The biexponential nature of the decays in BPH and BPHF is likely to be associated with a conformational change of the 
TABLE 1: Spectroscopic Data for the Studied Oligophenyl and Oligothienyl Swivel Cruciforms in Methylcyclohexane at Room Temperature (293 K), Low Temperature (77 K), and in the Solid State (Thin Films) ${ }^{a}$

\begin{tabular}{|c|c|c|c|c|c|c|c|c|c|c|c|}
\hline compound & $\begin{array}{c}\lambda_{\max }^{\mathrm{Abs}} \\
(\mathrm{nm}) \\
293 \mathrm{~K}\end{array}$ & $\begin{array}{l}\lambda_{\max }^{\mathrm{Abs}} \\
(\mathrm{nm}) \\
77 \mathrm{~K}\end{array}$ & $\begin{array}{l}\lambda_{\max }^{\mathrm{Abs}} \\
(\mathrm{nm}) \\
\text { film }\end{array}$ & $\begin{array}{c}\lambda_{\max }^{S_{0} \leftarrow S_{1}} \\
(\mathrm{~nm}) \\
293 \mathrm{~K}\end{array}$ & $\begin{array}{c}\lambda_{\max }^{S_{0}-S_{1}} \\
(\mathrm{~nm}) \\
77 \mathrm{~K}\end{array}$ & $\begin{array}{c}\lambda_{\max }^{S_{0} \leftarrow S_{1}} \\
\text { (nm) } \\
\text { film }\end{array}$ & $\begin{array}{c}\lambda_{\max }^{S_{0} \leftarrow T_{1}} \\
(\mathrm{~nm})\end{array}$ & $\begin{array}{l}\lambda_{\max }^{T_{1} \rightarrow T_{n}} \\
(\mathrm{~nm})^{c}\end{array}$ & $\begin{array}{c}\lambda_{\max }^{T_{1} \rightarrow T_{n}} \\
(\mathrm{~nm}) \\
\text { film }\end{array}$ & $\begin{array}{c}\epsilon_{\mathrm{TT}} \\
\left(\mathrm{M}^{-1} \mathrm{~cm}^{-1}\right)\end{array}$ & $\begin{array}{c}\text { Stokes } \\
\text { shift } \\
\left(\mathrm{cm}^{-1}\right)\end{array}$ \\
\hline $\mathrm{BPH}$ & $\begin{array}{c}277 \\
281^{b}\end{array}$ & 274 & $\begin{array}{r}280 \\
277^{b}\end{array}$ & $\begin{array}{r}396 \\
394^{b}\end{array}$ & 375 & $\begin{array}{l}392 \\
406^{b}\end{array}$ & 508 & $\begin{array}{l}480 \\
(480)\end{array}$ & 490 & 37010 & 10849 \\
\hline BPHF & 324 & 326 & 325 & 391 & $\frac{372}{391}$ & 401 & 555 & $\begin{array}{l}605 \\
(610)\end{array}$ & 615 & 26590 & 5289 \\
\hline BTF & 364 & 370 & 366 & 450 & $\begin{array}{l}428 \\
455\end{array}$ & 460 & 685 & $\begin{array}{l}600 \\
(570)\end{array}$ & 600 & 23500 & 5250 \\
\hline
\end{tabular}

${ }^{a}$ Underlined wavelengths is the maximum band. ${ }^{b}$ Data in $\mathrm{CHCl}_{3}$ and films (obtained by spin cast) taken from ref $1 .{ }^{c}$ In brackets, data is obtained from pulse-radiolysis spectra.

TABLE 2: Photophysical Parameters for the Studied Oligophenyl and Oligothienyl Swivel Cruciforms in Methylcyclohexane at Room Temperature (293 K), Low Temperature (77 K), and in Thin Films

\begin{tabular}{|c|c|c|c|c|c|c|c|c|c|c|c|c|c|}
\hline compound & $\begin{array}{c}\phi_{\mathbf{F}} \\
293 \mathrm{~K}\end{array}$ & $\begin{array}{c}\tau_{\mathbf{F}} \\
(\mathrm{ns})\end{array}$ & $\begin{array}{c}\phi_{\mathbf{F}} \\
77 \mathrm{~K}\end{array}$ & $\begin{array}{c}\phi_{\mathbf{F}} \\
\text { film }\end{array}$ & $\begin{array}{c}\tau_{\mathbf{F}} \\
(\mathrm{ns}) \\
\text { film }\end{array}$ & $\begin{array}{c}\phi_{\text {IC }} \\
293 \mathrm{~K}\end{array}$ & $\begin{array}{c}\phi_{\mathbf{P h}} \\
77 \mathrm{~K}\end{array}$ & $\begin{array}{c}\tau_{\mathbf{P h}} \\
(\mathrm{ms}) \\
77 \mathrm{~K}\end{array}$ & $\begin{array}{c}\phi_{\mathrm{T}} \\
293 \mathrm{~K}\end{array}$ & $\begin{array}{c}\phi_{\Delta} \\
293 \mathrm{~K}\end{array}$ & $\begin{array}{c}\tau_{\mathbf{T}} \\
(\mu \mathrm{s}) \\
293 \mathrm{~K}\end{array}$ & $\begin{array}{c}\text { triplet } \\
\text { energy } \\
(\mathrm{eV})\end{array}$ & $\begin{array}{c}\text { singlet } \\
\text { energy } \\
(\mathrm{eV})\end{array}$ \\
\hline $\mathrm{BPH}$ & $\begin{array}{l}0.18 \\
0.13^{a}\end{array}$ & 2.15 & $\begin{array}{r}\approx 0.60 \\
0.60\end{array}$ & $\begin{array}{l}0.064 \\
0.06^{a}\end{array}$ & 4.95 & 0.65 & 0.13 & 2461 & 0.17 & 0.18 & 11.1 & 2.76 & 3.79 \\
\hline BPHF & 0.87 & 1.11 & 0.63 & 0.41 & 1.62 & $\approx 0$ & 0.0019 & 0.00718 & 0.13 & 0.12 & 2.23 & $\begin{array}{c}2.58 \\
2.56 \pm 0.05^{c}\end{array}$ & 3.48 \\
\hline BTF & 0.078 & 0.34 & 0.054 & 0.079 & 2.42 & 0.49 & 0.0013 & 3.7 & 0.43 & 0.44 & 2.19 & 2.19 & 3.04 \\
\hline
\end{tabular}

${ }^{a}$ Data in $\mathrm{CHCl}_{3}$ and films (obtained by spin cast) taken from ref $1 .{ }^{b}$ Values taken from the intersection between the room temperature absorption and the fluorescence emission spectra. ${ }^{c}$ Value obtained from the energy transfer method, from pulse radiolysis measurements.

initially excited oligomer (shortest component) to a relaxed and more planar conformation (longest decay time). The crystal structure of BPH in the solid state has already been presented, ${ }^{1}$ and although this may not be identical to the solution behavior, NMR evidence has also been given to show the feasibility of such a relaxation in the excited state. Since the process observed in these biexponential decays is most likely to involve a conformational change, we can determine the rate constant for this $\left(k_{\text {conf }}\right)$ to be equal to $1 / \tau_{\text {short }}$. This leads to values (decay time values from Figure 2) of $k_{\text {conf }}=7.81 \times 10^{9} \mathrm{~s}^{-1}$ for BPH, $2.72 \times 10^{10} \mathrm{~s}^{-1}$ for BPHF, and $2.4 \times 10^{10} \mathrm{~s}^{-1}$ for BTF in $\mathrm{MCH}$.

A more detailed analysis of the decay profiles shows that, in the case of BPHF and BTF, the components associated with the rise time have lifetimes of $30-40 \mathrm{ps}$, whereas the decay components are longer (hundreds to thousands of picoseconds). It is worth noting that, with poly(9,9-di(ethylhexyl(fluorene)), ${ }^{20}$ a similar rise time (29 ps) is observed at long wavelengths and attributed to conformational relaxation, probably involving torsional changes around the $\mathrm{C}-\mathrm{C}$ bond between two fluorene rings. In the case of $\mathrm{BPH}$, the shorter component is longer $(\sim 128$ ps) which suggests a different mechanism for conformational change in the excited state. In fact, the absence of rising component in BPH suggests that possibly either a very fast conversion (shorter than the time resolutions of the system) is present or the overall mechanism related with this process involves rotation around the $\mathrm{C}-\mathrm{C}$ bond in the biphenyl unit leading to a slower process. In contrast with the two other cruciform oligomers, BPHF and BTF, this would involve rotation around the $\mathrm{C}-\mathrm{C}$ bond connecting the phenyl-fluorene (in BPHF) and thiophene-fluorene (in BTF) in each separated moiety. By analogy with previously studied $p$-phenylenevinylene oligomers, this would be consistent with the occurrence of conformational relaxation within the oligomer skeleton. ${ }^{12}$ Moreover, an additional observation, which seems to favor this hypothesis, is that with $\mathrm{BPH}$; the rotational change is made within the $\mathrm{C}-\mathrm{C}$ bond. $\mathrm{BPH}$ displays the longest decay time (1704 ps in $\mathrm{MCH}$ and $1540 \mathrm{ps}$ in $3 \mathrm{MP}$ ), while comparison with the literature data for $p$-terphenyl with a decay time of 1.3 ns $(1300 \mathrm{ps})^{15}$ strongly suggests that we are now observing the fluorescence lifetime relative to the $p$-terphenyl structure. In the cases of BPHF and BTF and on the basis of the decay times obtained (Figure 2), there is strong support for the involvement of the dimethyl-fluorene unit and benzyl and thiophene units in the decay of the relaxed species. In fact, if the dimethylfluorene unit would be uncoupled to the other units, a decay time of $6.1 \mathrm{~ns}$ (found for fluorene in $\mathrm{MCH}$ ) or $10 \mathrm{~ns}^{15}$ would be observed in the decay of BPHF and BTF. However, in the two cases, BPHF and BTF, the longest decay components are much shorter lived than the value for fluorene which clearly indicates coupling involving the benzyl and thiophene units. However, in the case of BTF, the 430 ps value is closed to the value found for the PF2/6 ( $\sim 370 \mathrm{ps)}$ ) and the rigid MeLPPP (300 ps) polymers. ${ }^{20}$ This strongly suggests different involvements of the dimethyl-fluorene unit within the cruciforms. The biexponential nature of the fluorescence decays in different fluorene oligomers with $n=2-6$ has been reported with an associated rise time. ${ }^{21}$ It is also interesting to note that the alkyl chain in the $9,9^{\prime}$ position strongly influences the decay time associated with fluorene oligomers. ${ }^{21-23}$ In the case of 9,9dihexylfluorene-2,2-7-diyl oligomers, the unimeric and trimeric compounds display lifetimes of 0.8 and $0.83 \mathrm{~ns}$, respectively, ${ }^{23}$ while with the 2-ethylhexyl group in the $9,9^{\prime}$ position, the dimer and trimer have lifetime values of 789 and 663 ps, respectively. ${ }^{22}$ These values are markedly shorter than the nonsubstituted alkyl oligomer. Moreover, the value for the decay time of BPHF (961 ps) is close to lifetimes reported for other alkyl substituted dimers or trimers, which seems to indicate that more than one fluorene unit is involved in the conjugation in the excited-state structure of BPHF.

The nature of the triple-exponential decay associated with BTF is also worthy of note. In the case of other alternating copolymers of thiophene and fluorene, multiexponential decays have been found. ${ }^{24,25}$ Triexponential decays have been attributed to a spectral diffusion of the emission spectra (shortest component) and the two other components (one with a 421 ps lifetime) have been attributed to emission from two different and localized sites within the polymer. ${ }^{24}$ The 421 ps component seems to correlate well with the 431 ps component (see Figure 2) found for BTF, suggesting the involvement of the thiophene and the fluorene units in this molecule. Although in the case of 

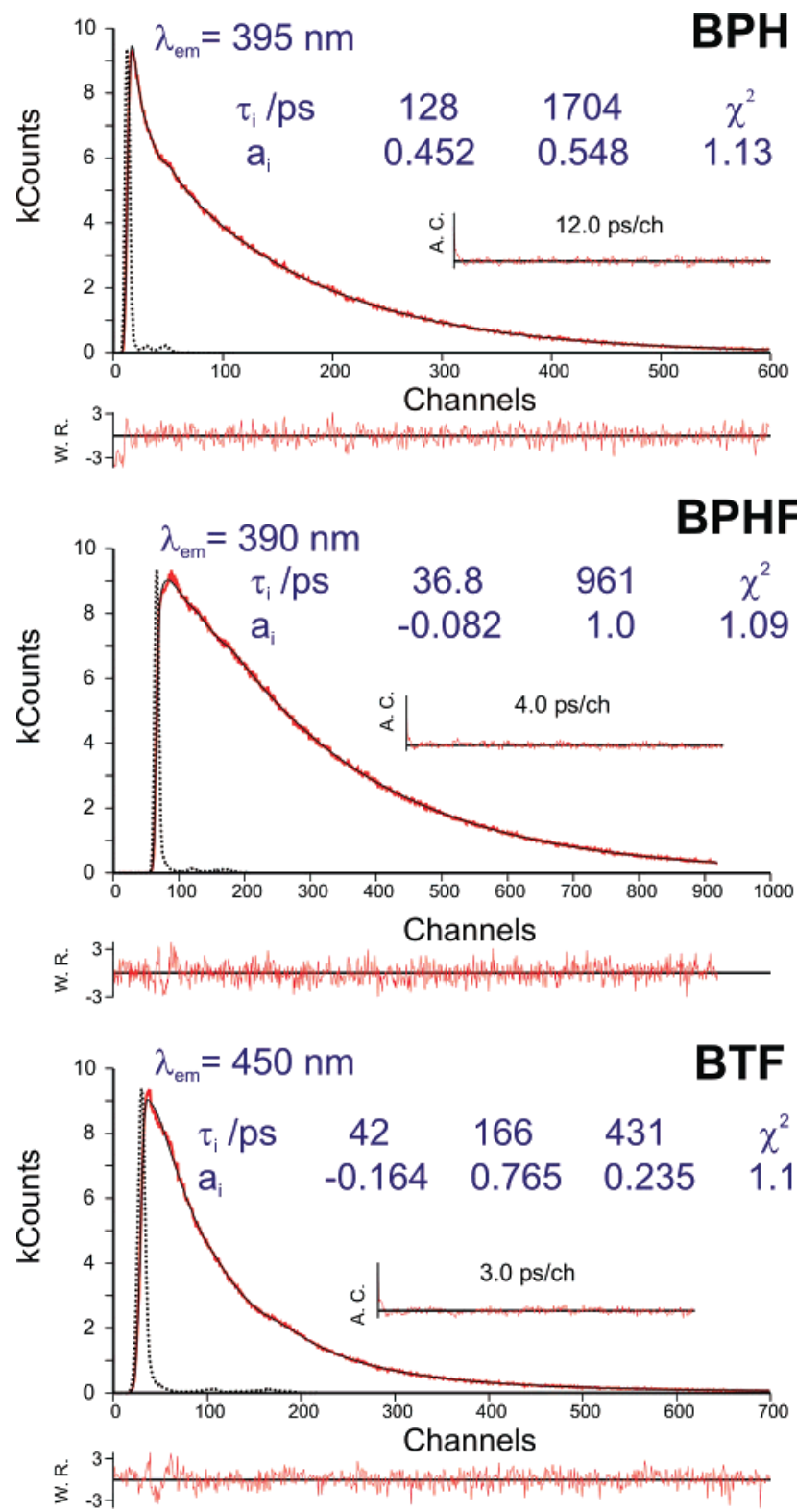

Figure 2. Fluorescence decays for BPH, BPHF, and BTF in methylcyclohexane obtained with $\lambda_{\mathrm{exc}}=302 \mathrm{~nm}$ and $T=293 \mathrm{~K}$. For a better judgment of the quality of the fits, autocorrelation functions (A.C.), weighted residuals (W.R.), and chi-square values $\left(\chi^{2}\right)$ are also presented as insets. The dashed lines in the decays are the pulse instrumental response.

the oligomer these "two localized sites" cannot be used in a direct comparison, they provide interesting elements for the understanding of the BTF decays. We attribute the nature of the three exponentials as follows. The first decay component (shortest) must be associated to the initial conformation of the BTF oligomer which then relaxes to two other independent conformations. The 166 ps component can then be related to the decay of a "species" involving some conjugation between the fluorene and the 3,3'-bithiophene, while the longest 431 ps component is likely to be related to a species involving the thiophene and fluorene units.

It is interesting to compare the values of the photophysical data for the fluorene containing compounds BPHF and BTF with those of fluorene itself. At room temperature in $\mathrm{MCH}$, fluorene has $\phi_{\mathrm{F}}=0.71$, whereas at low temperature, the value is $0.83 .{ }^{15}$ Whereas in the case of BPHF the $\phi_{\mathrm{F}}$ values are close to those of fluorene, in the case of BTF there is marked difference which shows the influence of the bithiophene unit
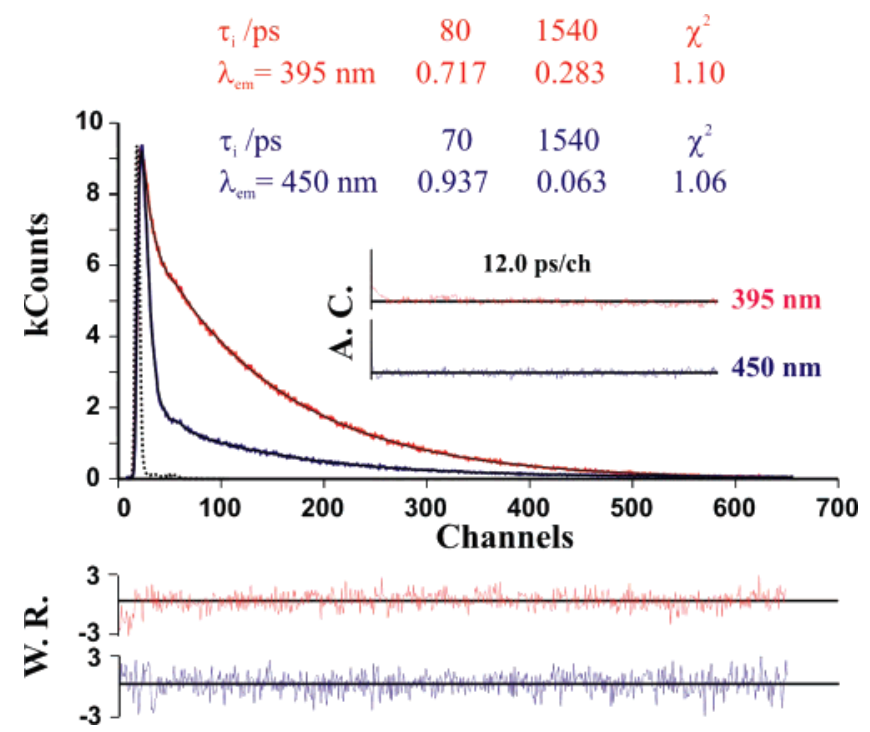

Figure 3. Fluorescence decays for BPH in 3-methylpentane obtained with $\lambda_{\text {exc }}=302 \mathrm{~nm}$ and at $T=293 \mathrm{~K}$. Shown as inset are the decay times obtained by independent analysis at each emission wavelength (395 and $450 \mathrm{~nm}$ ) together with the autocorrelation functions (A.C.), weighted residuals (W.R.), and chi-square values which attest to the goodness of fits.

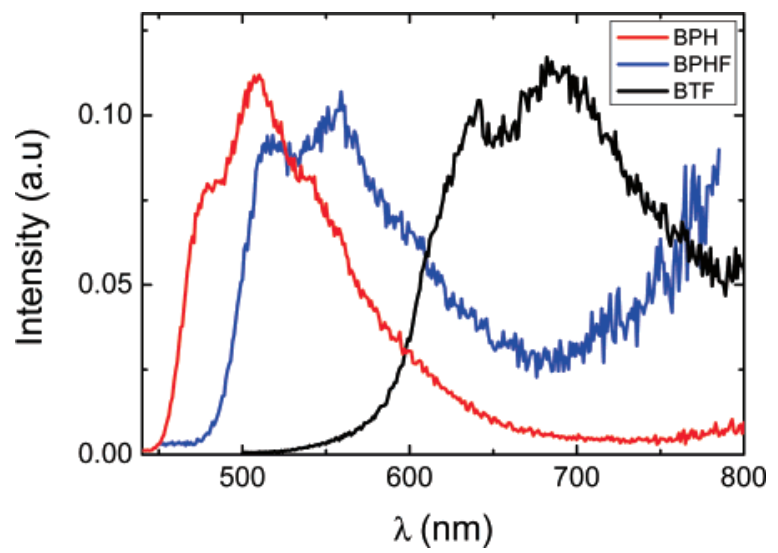

Figure 4. Phosphorescence emission spectra for the cruciforms in methylcyclohexane at $77 \mathrm{~K}$.

within the BTF. In fact, for 2,2'-bithiophene in methylcyclohexane, $\phi_{\mathrm{F}}=0.014,{ }^{8}$ which is very close to the value found for BTF in Table 2 and confirms the nature of the 166 ps component (with a pre-exponential factor of 0.765 ) in Figure 2.

Emission from the Triplet State. Phosphorescence spectra were recorded for the compounds in $\mathrm{MCH}$ glasses at $77 \mathrm{~K}$ and are shown in Figure 4. A vibrationally structured band in the $450-800 \mathrm{~nm}$ region is observed for all samples. This type of functionalization thus leads to emissive triplets, in contrast with related straight chain oligomers and polymers, which in general are not luminescent. There are, however, reports on phosphorescence of thiophene and thiophene-based oligomers. ${ }^{26-29}$

The triplet states of the copolymers were also characterized through transient absorption spectra in $\mathrm{MCH}$ solution at $293 \mathrm{~K}$ and in the solid state (thin films) (Figure 5). In addition to ground state depletion at shorter wavelengths, the spectra show intense absorption bands with maxima at longer wavelengths. In particular, with exception of BPH which exhibits a band with maxima at $490 \mathrm{~nm}$ and further absorption in the 300-600 nm region, the other compounds show sharp transient triplet-triplet absorption bands between 450 and $800 \mathrm{~nm}$. Comparison between the transient triplet-triplet absorption spectra in solution at 293 


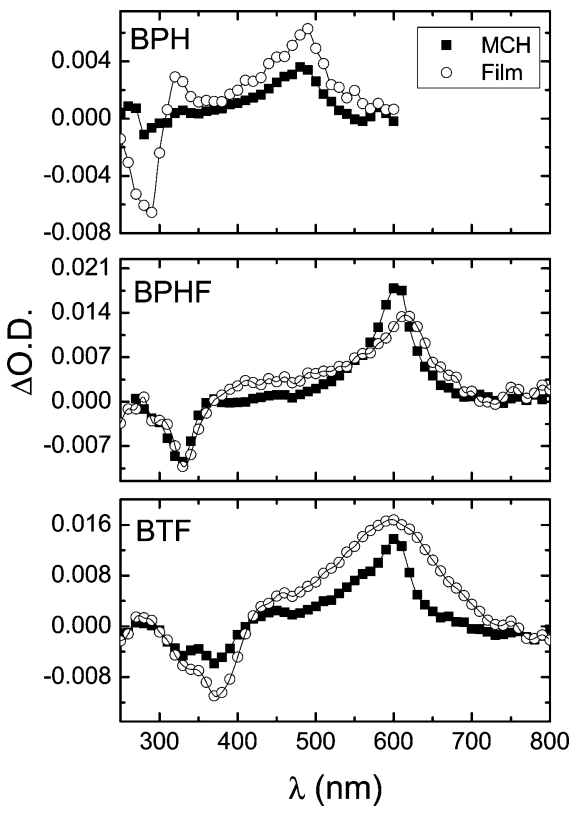

Figure 5. Transient triplet-triplet absorption spectra for the cruciform oligomers in methylcyclohexane solution and in thin films.

$\mathrm{K}$ and those in thin films indicates a red shift of approximately $10 \mathrm{~nm}$ in the absorption maxima. The exception was BTF, where no changes in the absorption maxima were observed when going from solution to the solid state.

Closer observation reveals very similar Stokes Shifts $(\sim 5200$ $\mathrm{cm}^{-1}$ ) for BPHF and BTF (Table 1). Although not negligible, this value seems to indicate that with these two compounds (BPHF and BTF) the differences in the potential energy curves of their $\mathrm{S}_{0}$ and $\mathrm{S}_{1}$ states are less marked than with $\mathrm{BPH}$, where there is a large difference between the maxima of fluorescence and the lowest absorption band, $10849 \mathrm{~cm}^{-1}$, see Table 1 . The differences observed with BPH imply either a significant structural difference between the potential energy curves of $S_{0}$ and $S_{1}$ states, which should be related to the possibility of rotation around the central unit (biphenyl) or that the emission is from a new species. Previous studies have implicated formation of an excited dimer to explain the nature of the emissive band and, consequently, the marked Stokes Shift. ${ }^{1}$ These interpretations rely upon X-ray crystallographic and NMR spectral data, which support formation of a folded helical conformation involving strong intramolecular $\pi-\pi$ interactions between the terminal phenyl rings adopted by BPH, both in the solid state and in solution. ${ }^{1}$ However, if such a $\pi$ dimer exists, it must be static since we have not been able to detect any rising component in our dynamic studies. Such a species should be seen dynamically as a rising component if the dimer is formed in the excited state (excimer). However, there is a possibility that it is produced and not observed as a rise time if superimposition of "monomer" and "excimer" emissions bands could cancel out the negative pre-exponential and thus omit the rising component in any part of the spectra.

In contrast, conformational changes resulting from rotation around the $\mathrm{C}-\mathrm{C}$ bond, leading to a more rigid and delocalized structure in the excited state, seem to be in agreement with the general behavior observed. This has been found with biphenyl in the $S_{0}$ and $S_{1}$ states and provides a reasonable explanation for the significant Stokes Shift observed in this case.

While the detailed explanation of the origin of this large Stokes Shift awaits further study, the large value observed, in which its is possible to eliminate reabsorption effects, makes
TABLE 3: Radiative $\left(k_{\mathrm{F}}\right)$ and Radiationless $\left(k_{\mathrm{NR}}, \mathrm{k}_{\mathrm{ISC}}\right.$, and $\left.k_{I C}\right)$ Rate Constants for the Studied Oligophenyl and Oligothienyl Swivel Cruciforms in MCH at Room Temperature $(293 \mathrm{~K})$

\begin{tabular}{lcccc}
\hline compound & $k_{\mathrm{F}}\left(\mathrm{ns}^{-1}\right)$ & $k_{\mathrm{NR}}\left(\mathrm{ns}^{-1}\right)$ & $k_{\mathrm{IC}}\left(\mathrm{ns}^{-1}\right)$ & $k_{\mathrm{ISC}}\left(\mathrm{ns}^{-1}\right)$ \\
\hline BPH & 0.084 & 0.381 & 0.30 & 0.079 \\
BPHF & 0.784 & 0.117 & $\approx 0$ & 0.117 \\
BTF & 0.229 & 2.71 & 1.44 & 1.26
\end{tabular}

this cruciform a good candidate for incorporation in either light emitting devices or polymeric lasers.

Photophysical Behavior. All of the photophysical parameters obtained for these cruciforms in solution and the solid state are presented in Table 2. For BPH and BTF, nonradiative deactivation quantum yields $\left(\phi_{\mathrm{IC}}+\phi_{\mathrm{T}}\right)$ indicate these are the principal pathways for excited-state deactivation. In contrast, with BPHF, the high fluorescence quantum yield (Table 2) shows that the radiative route is dominant. From this data, radiative and radiationless rate constants have been calculated for the three compounds in $\mathrm{MCH}$ solution at room temperature (Table 3). The radiative rate constant is dominant with $\mathrm{BPHF}$, whereas for $\mathrm{BPH}$ and $\mathrm{BTF}$ the nonradiative rate constant $\left(k_{\mathrm{NR}}\right)$ is faster and controls the decay of these two compounds. However, with $\mathrm{BPH}$, the radiationless $k_{\mathrm{IC}}$ rate constant is approximately four times higher than $k_{\mathrm{ISC}}$, whereas with $\mathrm{BTF}$, the two radiationless rate constants are approximately identical.

For $\mathrm{BPH}$, the fluorescence quantum yields obtained in solution and in films are in good agreement with the values previously reported in another solvent $\left(\mathrm{CHCl}_{3}\right)$ and in the solid state (films obtained by spin casting); see Table 1 and ref 1 .

As has been discussed earlier, with BTF, although the link in the bithienyl unit is through the 3,3'-position, the fluorescence quantum yield ( $\phi_{\mathrm{F}}=0.078$, see Table 2$)$ is of a similar order of magnitude to that of $2,2^{\prime}$-bithiophene $\left(\phi_{\mathrm{F}}=0.014\right.$ in methylcyclohexane solution), ${ }^{8}$ suggesting that the main conjugation unit for radiative deactivation is associated with the bithiophene moiety rather than coupling between the thienyl and the fluorene units.

For BPHF and BTF, the low phosphorescence quantum yields and the high intersystem-crossing $\left(\phi_{\mathrm{T}}\right)$ values indicate that nonradiative channels are the main routes for the triplet state decay. In contrast, with $\mathrm{BPH}$, the $\phi_{\mathrm{Ph}}$ value is close to the $\phi_{\mathrm{T}}$ value, showing that the triplet state decay is predominantly radiative.

The large differences between the $\tau_{\mathrm{Ph}}$ values obtained for the three compounds are worth noting, with BPH presenting a $\tau_{\mathrm{Ph}}$ value of $2.46 \mathrm{~s}$, whereas for $\mathrm{BTF}$, the value is reduced to 3.7 $\mathrm{ms}$, and for BPHF, it is $7.18 \mu \mathrm{s}$; see Table 2. This strongly suggests different natures of the emissive triplet states, with the lowest lying triplet state changing with both the nature of the central unit (bridging the two oligomer units) and also the nature of the sidearm of the cruciform. Comparison between BPH and BPHF shows that substitution of the $t$-butyl-benzyl sidearm by the 1,1-dimethylfluorene decreases the forbidden nature of the $\mathrm{T}_{1} \rightarrow \mathrm{S}_{0}$ transition. The substitution of the central biphenyl unit by the 3,3'-bithiophene unit leads to an increase of the $\tau_{\mathrm{Ph}}$ value by a factor of 1000 indicating the potential involvement of the $n$ orbitals of the sulfur atoms in the $\mathrm{T}_{1}$ state. It is also useful to compare the triplet data for the fluorene containing compounds (BPHF and BTF) with the triplet state emissive properties of fluorene $\left(\phi_{\mathrm{Ph}}=0.04\right.$, or 0.07 in a polar solvent, ${ }^{15}$ and $\tau_{\mathrm{Ph}}=$ $5.1 \mathrm{~s}$ in a nonpolar solvent ${ }^{15}$ and $\tau_{\mathrm{Ph}}=5.7 \mathrm{~s}$ in a polar solvent). ${ }^{15}$ The phosphorescence quantum yields for BPHF and BTF are an order of magnitude lower than that for fluorene, and even more dramatic decreases are seen in the phosphorescence 
lifetimes, which are three (BTF) and six (BPHF) orders of magnitude lower than the parent compound. This shows the strong involvement of the 1,4-phenylene and thiophene groups in the triplet state of these compounds. In addition, it is possible with the cruciforms that conformational processes are involved in the fast nonradiative deactivation of BTF and BPHF.

The triplet states of the cruciforms have also been investigated by pulse radiolysis of nitrogen saturated benzene solutions using biphenyl as sensitizer. Similar transient absorption bands were observed for these oligomers to those seen in laser flash photolysis, see $\lambda_{\max }^{T_{1} \rightarrow T_{n}}$ in Table 1 .

For comparison with phosphorescence data and for validation of the pulse radiolysis-energy transfer method, ${ }^{30}$ the triplet state energy of BPHF was also studied by sensitization using various energy donors following pulse radiolysis of benzene solutions. ${ }^{31}$ Triplet sate formation of BPHF was not observed when acridine (1.97 eV), 9-fluorenone (2.19 eV), or benzil $(2.31 \mathrm{eV})$ were used as sensitizers showing that the triplet energy of this cruciform lies above these. ${ }^{15}$ With chrysene $(2.48 \mathrm{eV}),{ }^{15}$ we were unable to induce the BPHF triplet state, but in this particular case, analysis was difficult because the transient absorption spectra of these two samples are very close. Sensitization of the triplet state of BPHF was achieved with naphthalene $(2.62 \mathrm{eV}),{ }^{15}$ showing that the triplet energy of the cruciform should lay between those of naphthalene and chrysene. This leads to a triplet energy of BPHF in solution at room temperature of $2.56 \pm 0.05 \mathrm{eV}$, in excellent agreement with the value taken from the onset of phosphorescence (ca. $480 \mathrm{~nm}$, $2.58 \mathrm{eV})$.

Using both pulse radiolysis-energy transfer and phosphorescence onset values, we find that it is possible to obtain a clear location of the triplet states of these compounds, as seen in Table 2 and Figure 3. From these and the singlet energies, it is possible to calculate the $S_{1}-T_{1}$ splittings, which give a measure of the electron-electron exchange energy. The $\Delta E_{\mathrm{S}_{1}-\mathrm{T}_{1}}$ values obtained $(0.92 \pm 0.08 \mathrm{eV})$ are very close to those seen with other conjugated polymers $\left(0.77 \pm 0.17 \mathrm{eV},{ }^{32} 0.7 \pm 0.1 \mathrm{eV}^{33}\right)$. Although the difference is close to the experimental error, the fact that the value is slightly larger for the cruciform oligomers is in agreement with the predicted ${ }^{33}$ and observed ${ }^{34}$ effect of conjugation length on the singlet-triplet splitting. In addition, it can be also be seen from Table 2 that this energy difference, $\Delta E_{\mathrm{S}_{1}-\mathrm{T}_{1}}$, decreases in the order BPH $(1.03 \mathrm{eV})$, BPHF $(0.90$ $\mathrm{eV})$, BTF $(0.85 \mathrm{eV})$. Application of the energy gap law for radiationless processes ${ }^{35}$ provides a ready explanation for the increasing importance of intersystem crossing on going from $\mathrm{BPH}$ to BPHF and BTF. However, with this latter compound, as with polythiophenes, ${ }^{36}$ extensive spin-orbit coupling from the sulfur atom may also play a role in making the $S_{1} \sim \sim T_{1}$ intersystem crossing process highly efficient, with a yield of 0.43 (see Table 2).

Singlet oxygen has been detected by its characteristic phosphorescence following photolysis of aerated solutions of the cruciforms. The yield of singlet oxygen formation $\left(\phi_{\Delta}\right)$ was determined by measuring the initial phosphorescence intensity at $1270 \mathrm{~nm}$ as a function of laser intensity and by comparing the slope with that for $1 H$-Phenalen-1-one or biphenyl as standards. From Table 2, it can be seen that the values for $\phi_{\Delta}$ and $\phi_{\mathrm{T}}$ are quite similar indicating that these cruciform oligomers sensitize singlet oxygen formation with near unit efficiency $\left(S_{\Delta}\right.$ $\sim 1)$. This is similar to the behavior observed with a wide range of conjugated polymers, ${ }^{36}$ and provides strong support for the measured values of intersystem crossing quantum yields.

\section{Summary/Conclusions}

We have obtained detailed spectroscopic and photophysical data which have allowed the characterization of three cruciforms in solution at room temperature, in low-temperature glasses and in thin Zeonex films. The structure is aimed at favoring formation of amorphous structures by hindering crystallization. From the fluorescence quantum yields of BPHF and BTF in solution, low-temperature glass, and thin film (Table 2), this appears to have been partially successful. A particularly high fluorescence quantum yield is obtained with the fluorenephenylene derivative BPHF. Fluorescence decays were biexponential for $\mathrm{BPH}$ and $\mathrm{BPHF}$ and triexponential for BTF. This is probably associated with conformational changes in the excited state, involving rotation about the cruciform carboncarbon bond.

Interesting results are observed with $\mathrm{BPH}$ where the fluorescence quantum yield in a glass at $77 \mathrm{~K}$ is in fact greater than that in solution at room temperature. It is also relevant that the value in the low-temperature glass $(0.60)$ is comparable to that of corresponding single chain molecule $p$-terphenyl in nonpolar solvents (0.77). ${ }^{15}$ A possible explanation is that internal conversion is favored at room temperature (Table 3) through rotations around the cruciform carbon-carbon bond, but this is frozen out at low temperatures.

In contrast to many other conjugated oligomers and polymers, all three derivatives are phosphorescent. With BTF, intersystem crossing is a particularly important decay pathway for the excited singlet state. This is probably associated with both a heavy atom effect of the sulfur and a relatively small $\mathrm{S}_{1}-\mathrm{T}_{1}$ splitting.

Acknowledgment. Financial support from FEDER and FCT (through Project Nos. POCI/QUI/55672/2004 and POCI/QUI/ 58291/2004) is acknowledged. J.P. acknowledges FCT for a Ph.D. grant (SFRH/BD/18876/2004). Pulse radiolysis experiments were carried out at the Free Radical Research Facility in the Synchrotron Radiation Department of the CLRC Daresbury Laboratory, Warrington, U.K., with the support of the European Commission through the "Improving Human Potential" Transnational Access to Major Research Infrastructures Contract HPRI-CT-2002-00183. Drs. S. Navaratnam and R. Edge (FRRF, Daresbury Laboratory) are acknowledged for their excellent technical support in these. J.S.d.M. and J.P. acknowledge the generous assistance of Prof. A. Maçanita (IST-UTL) for the use of his ps-TCSPC for the measurement of the fluorescence decays with picosecond time resolution.

Supporting Information Available: Synthesis and characterization of the cruciform dimers BTF and BPHF. This material is available free of charge via the Internet at http://pubs.acs.org.

\section{References and Notes}

(1) Nehls, B. S.; Galbrecht, F.; Bilge, A.; Brauer, D. J.; Lehmann, C. W.; Scherf, U.; Farrell, T. Org. Biomol. Chem. 2005, 3, 3213.

(2) Mullen, K., Wegner, G., Eds. Electronic Materials-The Oligomer Approach; Wiley-VCH: Weinheim, 1998.

(3) Wang, H. Y.; Wan, J. H.; Jiang, H. J.; Wen, G. A.; Feng, J. C.; Zhang, Z. J.; Peng, B.; Huang, W.; Wei, W. J. Polym. Sci., Part A: Polym. Chem. 2007, 45, 1066.

(4) Wilson, J. N.; Smith, M. D.; Enkelmann, V.; Bunz, U. H. F. Chem. Commun. 2004, 1700 .

(5) Wilson, J. N.; Josowicz, M.; Wang, Y. Q.; Bunz, U. H. F. Chem. Commun. 2003, 2962

(6) Bilge, A.; Zen, A.; Forster, M.; Li, H. B.; Galbrecht, F.; Nehls, B. S.; Farrell, T.; Neher, D.; Scherf, U. J. Mater. Chem. 2006, 16, 3177.

(7) Saragi, T. P. I.; Fuhrmann-Lieker, T.; Salbeck, J. Synth. Met. 2005, $148,267$. 
(8) Becker, R. S.; Seixas de Melo, J.; Maçanita, A. L.; Elisei, F. J. Phys. Chem. 1996, 100, 18683

(9) Pina, J.; Seixas de Melo, J.; Burrows, H. D.; Bilge, A.; Farrell, T.; Forster, M.; Scherf, U. J. Phys. Chem. B 2006, 110, 15100.

(10) Lima, J. C.; Abreu, I.; Brouillard, R.; Maçanita, A. L. Chem. Phys. Lett. 1998, 298, 189.

(11) Giestas, L.; Yihwa, C.; Lima, J. C.; Vautier-Giongo, C.; Lopes, A.; Maçanita, A. L.; Quina, F. H. J. Phys. Chem. A 2003, 107, 3263.

(12) Di Paolo, R. E.; Seixas de Melo, J.; Pina, J.; Burrows, H. D.; Morgado, J.; Maçanita, A. L. ChemPhysChem 2007, 8, 2657-2664.

(13) Striker, G.; Subramaniam, V.; Seidel, C. A. M.; Volkmer, A. J. Phys. Chem. B 1999, 103, 8612.

(14) Bensasson, R. V.; Land, E. J.; Truscott, T. G. Excited states and free radicals in biology and medicine; Oxford Science Publications: Oxford, 1993.

(15) Murov, S.; Charmichael, I.; Hug, G. L. Handbook of Photochemistry, 2nd ed.; Dekker Inc.: New York, 1993.

(16) Kumar, C. V.; Qin, L.; Das, P. K. J. Chem. Soc., Faraday Trans. $2 \mathbf{1 9 8 4}, 80,783$

(17) Seixas de Melo, J.; Silva, L. M.; Kuroda, M. J. Chem. Phys. 2001, 115,5625

(18) deMello, J. C.; Wittmann, H. F.; Friend, R. H. Adv. Mater. 1997, 9, 230 .

(19) Palsson, L. O.; Monkman, A. P. Adv. Mater. 2002, 14, 757.

(20) Dias, F. B.; Maçanita, A. L.; Seixas de Melo, J.; Burrows, H. D.; Guntner, R.; Scherf, U.; Monkman, A. P. J. Chem. Phys. 2003, 118, 7119

(21) Anemian, R.; Mulatier, J. C.; Andraud, C.; Stephan, O.; Vial, J. C. Chem. Commun. 2002, 1608.
(22) Chi, C. Y.; Im, C.; Wegner, G. J. Chem. Phys. 2006124.

(23) Wasserberg, D.; Dudek, S. P.; Meskers, S. C. J.; Janssen, R. A. J. Chem. Phys. Lett. 2005, 411, 273.

(24) Charas, A.; Morgado, J.; Alcacer, L.; Martinho, J. M. G.; Cacialli, F. Synth. Met. 2003, 135, 387 .

(25) Morgado, J.; Charas, A.; Martinho, J. M. G.; Alcacer, L. Synth. Met. 2005, 154, 81 .

(26) Wasserberg, D.; Marsal, P.; Meskers, S. C. J.; Janssen, R. A. J.; Beljonne, D. J. Phys. Chem. B 2005, 109, 4410.

(27) Wasserberg, D.; Meskers, S. C. J.; Janssen, R. A. J.; Mena-Osteritz, E.; Bauerle, P. J. Am. Chem. Soc. 2006, 128, 17007.

(28) Rentsch, S.; Yang, J. P.; Paa, W.; Birckner, E.; Schiedt, J.; Weinkauf, R. Phys. Chem. Chem. Phys. 1999, 1, 1707.

(29) Pina, J.; Burrows, H. D.; Becker, R. S.; Dias, F. B.; Maçanita, A. L.; Seixas de Melo, J. J. Phys. Chem. B 2006, 110, 6499.

(30) Monkman, A. P.; Burrows, H. D.; Miguel, M. D.; Hamblett, I.; Navaratnam, S. Synth. Met. 2001, 116, 75.

(31) Burrows, H. D.; Seixas de Melo, J.; Serpa, C.; Arnaut, L. G.; Miguel, M. D.; Monkman, A. P.; Hamblett, I.; Navaratnam, S. Chem. Phys. 2002, 285, 3 .

(32) Monkman, A. P.; Burrows, H. D.; Hartwell, L. J.; Horsburgh, L. E.; Hamblett, I.; Navaratnam, S. Phys. Rev. Lett. 2001, 86, 1358.

(33) Kohler, A.; Beljonne, D. Adv. Funct. Mater. 2004, 14, 11.

(34) Monkman, A.; Burrows, D. Synth. Met. 2004, 141, 81

(35) Englman, R.; Jortner, J. Mol. Phys. 1970, 18, 145.

(36) Burrows, H. D.; Seixas de Melo, J.; Serpa, C.; Arnaut, L. G.; Monkman, A. P.; Hamblett, I.; Navaratnam, S. J. Chem. Phys. 2001, 115, 9601. 